\title{
Numerical implementation of a modified Mohr-Coulomb model and its application in slope stability analysis
}

\author{
Xu Xiang ${ }^{1} \cdot$ Dai Zi-Hang ${ }^{2}$
}

Received: 25 July 2016/Revised: 11 January 2017/Accepted: 15 January 2017/Published online: 6 March 2017

(C) The Author(s) 2017. This article is published with open access at Springerlink.com

\begin{abstract}
The hyperbolic function proposed by AbboSloan was employed not only to approach the MohrCoulomb criterion but also to express the plastic potential function. A better approximation to the Mohr-Coulomb yield and potential surfaces was achieved by increasing the transition angle and proven to be highly efficient in numerical convergence. When a Gaussian integral point goes into plastic state, two cases on yield stress adjustments were introduced. They may avoid solving the second derivative of the plastic potential function and the inverse matrix compared with the existing subroutine. Based on the above approaches, a fully implicit backward Euler integral regression algorithm was adopted. The two- and three-dimensional user subroutines which can consider the associated or non-associated flow rule were developed on the platform of the finite element program-ABAQUS. To verify the reliability of these two subroutines, firstly, the numerical simulations of the indoor conventional triaxial compression and uniaxial tensile tests were performed, and their results were compared with those of the embedded Mohr-Coulomb model and the analytical approach. Then the main influential factors including the associated or nonassociated flow rule, the judgment criteria of slope failure, and the tensile strength of soil were analyzed, and the application of the two-dimensional subroutine in the stability analysis of a typical soil slope was discussed in detail
\end{abstract}

Dai Zi-Hang

dzhang@fzu.edu.cn

1 College of Transportation and Civil Engineering, Fujian Agriculture and Forestry University, Fuzhou 350108, People's Republic of China

2 College of Civil Engineering, Fuzhou University, Fuzhou 350108, People's Republic of China through comparisons with the embedded model and the limit analysis method, which shows that this subroutine is more applicable and reliable than the latter two.

Keywords Hyperbolic yield function - Plastic potential function - First derivative - Stress adjustment - Slope stability analysis

\section{Introduction}

In 1773, Coulomb proposed a soil pressure theory of soil or rock failure, which is expressed by

$\tau=c-\sigma \tan \phi$,

where $\tau$ and $\sigma$ are respectively the shear strength and the normal stress (tensile stress is positive) in the shearing surface; $c$ and $\phi$ are the cohesion and the angle of internal friction of soil or rock, respectively.

Later, Mohr developed the Coulomb failure condition into the law of shear failure, namely the Mohr-Coulomb (M-C for short hereinafter) yield criterion, that could be described in terms of the principal stresses $\left(\sigma_{1} \geq \sigma_{2} \geq \sigma_{3}\right)$ as

$F=\left(\sigma_{1}-\sigma_{3}\right)+\left(\sigma_{1}+\sigma_{3}\right) \sin \phi-2 c \cos \phi=0$.

A large number of experiments have shown that the M$\mathrm{C}$ yield criterion is able to reasonably depict the yield or failure behavior of soil and rock. In addition to Eq. (2), the $\mathrm{M}-\mathrm{C}$ criterion can be expressed in many other forms, for example the following one in terms of stress invariants [1]:

$F=\sigma_{\mathrm{m}} \sin \phi+\bar{\sigma}\left(\cos \theta-\frac{1}{\sqrt{3}} \sin \phi \sin \theta\right)-c \cos \phi=0$, 
where $\sigma_{\mathrm{m}}$ is the average value of the three principal stresses; $\bar{\sigma}=\sqrt{J_{2}}$, which is the equivalent stress; $J_{2}$ is the second deviatoric stress invariant; and $\theta$ is the Lode angle ranging from $-30^{\circ}$ to $30^{\circ}$.

It is well known that the $\mathrm{M}-\mathrm{C}$ yield criterion expresses a hexagonal pyramid surface in principal stress space, which has a flaw in numerical computation, i.e., the gradient discontinuities which occur at both the edges and the tip of the hexagonal pyramid surface. For that, researchers have ever presented the following three kinds of methods to overcome the flaw. Firstly, Drucker-Prager proposed the smooth circular conical surfaces, including the circumscribed, inscribed, and middle ones [2, 3], such that the ideal elasto-plastic constitutive model based on the Drucker-Prager criterion was one of the earliest models used for soil and rock and was embedded in some of the earliest commercial finite element programs, such as SAP, ADINA, ANSYS. Although the above circular conical surfaces overcome the numerical singularity of the original $\mathrm{M}-\mathrm{C}$ model, they are significantly different in geometrical shape from the hexagonal pyramid of the M-C model, resulting in the advent of more or less errors [3] even if the equal area cone $[4,5]$ is employed while the above-mentioned programs are used. Secondly, the original $\mathrm{M}-\mathrm{C}$ surface is viewed as six separate planar yield surfaces and the constitutive law is implemented as a multi-surface yield function using the formulation of Koiter [6] and Clausen et al. [7]. The third kind of methods, which is employed in this paper, is smoothing the vertices and approaching the $\mathrm{M}-\mathrm{C}$ yield surface.

In order to sufficiently approach the original $\mathrm{M}-\mathrm{C}$ yield surface, Abbo-Sloan [8] proposed a simple hyperbolic yield surface to eliminate the singularity of the $\mathrm{M}-\mathrm{C}$ yield surface and presented two efficient FORTRAN 77 subroutines to illustrate how this yield surface is implemented in practice. However, they did not build an entire numerical constitutive model. Based on Abbo-Sloan function, a userdefined material subroutine (UMAT) was developed on the platform of ABAQUS by Jia et al. [9] using FORTRAN language.

Generally speaking, the M-C model can be reliably applied to stability analysis in geotechnical problems such as earth pressure [10, 11], slope stability [12, 13], and bearing capacity of foundation soil $[14,15]$. ABAQUS is a well-known nonlinear finite element software, in which actually an embedded $\mathrm{M}-\mathrm{C}$ model is included. And in the embedded $\mathrm{M}-\mathrm{C}$ model the true $\mathrm{M}-\mathrm{C}$ yield surface is used but the plastic potential function proposed by MenétreyWilliam [16] is employed, which presents a hyperbola in meridional plane and a closed smooth curve combined by three elliptic arcs in deviatoric plane, and it is the very inconsistency between the yield and potential functions so that the plastic flow rule of the model is always non-associated. Furthermore, this inconsistency is also a theoretical flaw of the embedded $\mathrm{M}-\mathrm{C}$ model in ABAQUS. Jia's UMAT model [9] has overcome this drawback, but it involves the second derivative of the plastic potential function, and the matrix inversion, resulting in not only the complexity of programming, but also the increases of the amount of calculation and the difficulty in numerical convergence, particularly when smoothed transition points are too close to the edges of the hexagonal pyramid surface. For that, a simplified method and the programming of new user-defined material subroutines will be introduced in this paper, and note that in the following text, UMAT only refers to the new subroutines. And in order to test and verify the UMAT, firstly, the indoor conventional triaxial compression and uniaxial tensile tests were numerically replicated with the UMAT and compared with the results of the embedded M-C model and analytical or Mohr circledrawing approaches. Then a typical two-dimensional homogeneous soil slope [17] was simulated with the UMAT and compared in detail with the results of the embedded $\mathrm{M}-\mathrm{C}$ model.

\section{Brief introduction of Abbo-Sloan yield function}

The hyperbolic yield function $F$ proposed by Abbo-Sloan [8] is smooth in both meridional and deviatoric planes and can be expressed as

$F=\sigma_{\mathrm{m}} \sin \phi+\sqrt{\bar{\sigma}^{2} K^{2}(\theta)+a^{2} c^{2} \cos ^{2} \phi}-c \cos \phi=0$,

where

$K(\theta)=\left\{\begin{array}{ll}A-B \sin 3 \theta & |\theta|>\theta_{\mathrm{T}} \\ \cos \theta-\frac{1}{\sqrt{3}} \sin \phi \sin \theta & |\theta| \leq \theta_{\mathrm{T}}\end{array}\right.$,

where

$$
\begin{aligned}
A= & \frac{1}{3} \cos \theta_{\mathrm{T}}\left[3+\tan \theta_{\mathrm{T}} \tan 3 \theta_{\mathrm{T}}+\right. \\
& \left.\frac{1}{\sqrt{3}} \times \operatorname{sign}(\theta)\left(\tan 3 \theta_{\mathrm{T}}-3 \tan \theta_{\mathrm{T}}\right) \sin \phi\right], \\
B= & \frac{1}{3 \cos 3 \theta_{\mathrm{T}}}\left(\operatorname{sign}(\theta) \sin \theta_{\mathrm{T}}+\frac{1}{\sqrt{3}} \cos \theta_{\mathrm{T}} \sin \phi\right),
\end{aligned}
$$

where

$\operatorname{sign}(\theta)=\left\{\begin{array}{cc}+1 & \theta \geq 0^{\circ} \\ -1 & \theta<0^{\circ}\end{array}\right.$ 
in above relevant expressions, $K(\theta)$ is used to control the shape of the yield surface in deviatoric plane; $\theta_{\mathrm{T}}$ is the transition angle ranging from $0^{\circ}$ to $30^{\circ}$, and the greater the value of $\theta_{\mathrm{T}}$, the better fitting the $\mathrm{M}-\mathrm{C}$ cross section in deviatoric plane, but $\theta_{\mathrm{T}}$ should not be too close to $30^{\circ}$ in order to avoid ill-conditioning of the approximation. A typical value of $\theta_{\mathrm{T}}$ is $25^{\circ}$ as suggested by Abbo-Sloan [8] and Jia et al. [9]. In Ref. [8], the negative branch of the hyperbola has been chosen, which takes into account the tensile strength of soil through parameter $a$. In fact, parameter $a$ is somewhat similar to the meridional eccentricity $\varepsilon$ included in the potential function of the embedded $\mathrm{M}-\mathrm{C}$ model [18], and the Abbo-Sloan yield function (Eq. 4) can closely model the M-C yield function (Eq. 3) by changing the magnitude of $a$ and will theoretically be degraded as the latter if $a$ is zero. The M-C yield surface and some sections of various smooth hyperbolic surfaces in a deviatoric plane are plotted in Fig. 1. Evidently, with the decrease of parameter $a$, the Abbo-Sloan yield surface gradually approaches the $\mathrm{M}-\mathrm{C}$ yield surface. However, to avoid the ill-conditioning of numerical calculation, $a$ is not allowed to be zero just as there is a limitation of the allowable minimum of 0.1 for the meridional eccentricity $\varepsilon$ in the embedded $\mathrm{M}-\mathrm{C}$ model [18]. Several meridional sections of the hyperbolic yield surfaces with different $a$ values as well as $\theta=0^{\circ}$ and $\phi=30^{\circ}$ are plotted in Fig. 2 [8]. One can infer that the hyperboloid is an approximation to the $\mathrm{M}-\mathrm{C}$ yield surface in the meridional plane. Obviously, $a$ value has a significant influence on the shape of the hyperbolic surface. When $a=0.05$ and $\theta_{\mathrm{T}}=25^{\circ}$, the hyperbolic surface almost approaches the $\mathrm{M}-\mathrm{C}$ yield surface with the maximum difference of less than $0.15 \%$ [8].

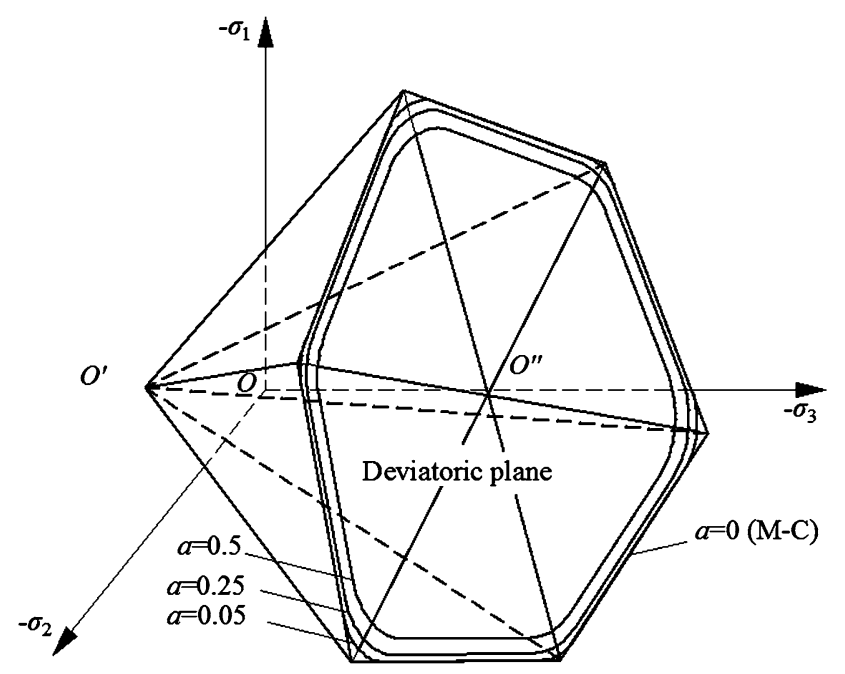

Fig. 1 M-C yield surface in 3D principal stress space and Abbo's rounded yield surface in deviatoric plane
From Fig. 2, it is shown that for a specific value of $a$, error always becomes less and less with the increase of the minor principle stress toward the direction of compression stress. Meanwhile, if assuming $\theta_{\mathrm{T}}=29^{\circ}$, as suggested by Owen and Hinton [19], the closer results to smooth the edges of the $\mathrm{M}-\mathrm{C}$ hexagonal yield surface pyramid will be obtained. And the examples in this paper will show that fast convergence can still be achieved and the higher precisions than those of Abbo-Sloan [8] and Jia et al. [9] can be obtained as well.

Theoretically, making the potential function similar to the yield function is reasonable, and only by this way, the associated flow rule can be achieved possibly. In this paper, the potential function $G$ was assumed to have the same form as the hyperbolic yield function $F$. The only difference is that the angle of internal friction $\phi$ was replaced with the dilation angle $\psi$ (always $\psi \leq \phi$ ). If $\psi=\phi$, the associated flow rule is adopted, otherwise the non-associated flow rule is used. Thus, the mathematical expression of $G$ is

$G=\sigma_{\mathrm{m}} \sin \psi+\sqrt{\bar{\sigma}^{2} K^{2}(\theta)+a^{2} c^{2} \cos ^{2} \psi}-c \cos \psi=0$.

Obviously, the geometrical shape of the potential surface is similar to the yield surface in Fig. 1, but the inclination angle (dilation angle) in the strain meridional plane (similar to the stress meridional plane shown in Fig. 2) is always less than or equal to the angle of internal friction. Note that for an ideal elasto-plastic model, the yield surface is fixed but the plastic potential surface may move outwards with the increase of plastic strain.

\section{First derivative used in UMAT}

Differentiation of the yield and potential functions with respect to stress may respectively obtain the flow vectors $\boldsymbol{n}$ and $\boldsymbol{n}^{\prime}$, which are used for the stress adjustment after

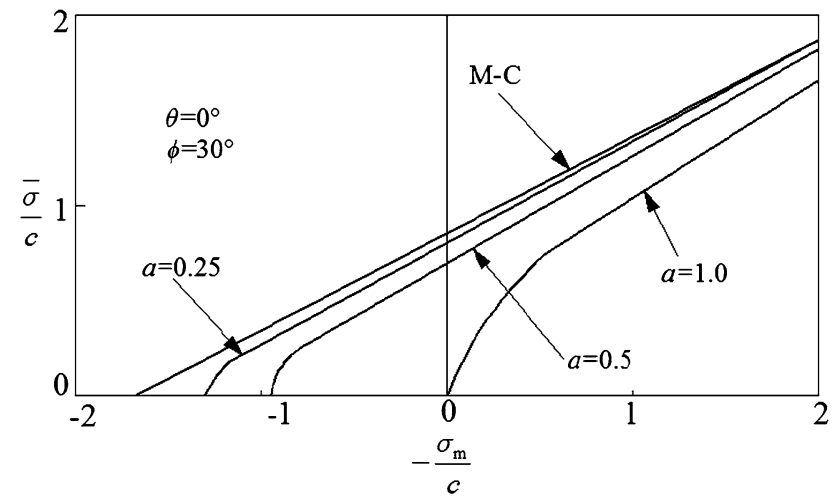

Fig. 2 Abbo's hyperbolic approximation to $\mathrm{M}-\mathrm{C}$ meridional section [8] 


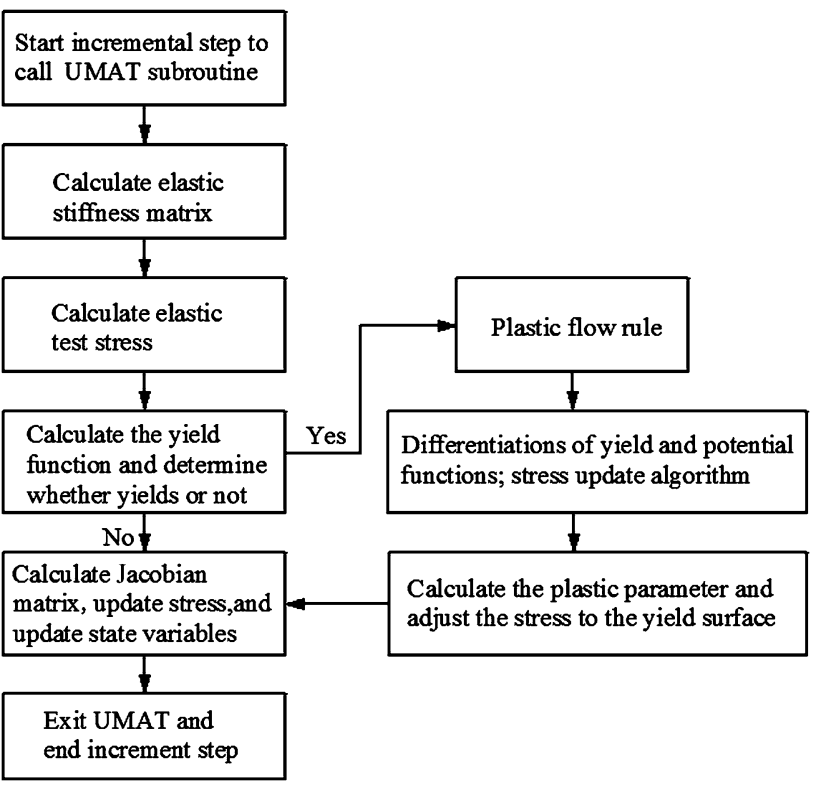

Fig. 3 Flowchart of the UMAT

yielding and are consistent with Refs. [8, 9, 20]; for example, the flow vector $\boldsymbol{n}$ is defined as

$\boldsymbol{n}=\frac{\partial F}{\partial \sigma}=C_{1} \frac{\partial \sigma_{\mathrm{m}}}{\partial \sigma}+C_{2} \frac{\partial \bar{\sigma}}{\partial \sigma}+C_{3} \frac{\partial J_{3}}{\partial \sigma}$,

where

$C_{1}=\sin \phi, C_{2}=\alpha\left(K-\tan 3 \theta \frac{\mathrm{d} K}{\mathrm{~d} \theta}\right)$,

$C_{3}=\alpha\left(-\frac{\sqrt{3}}{2 \cos 3 \theta \bar{\sigma}^{2}} \frac{\mathrm{d} K}{\mathrm{~d} \theta}\right)$,

$\alpha=\frac{\bar{\sigma} K}{\sqrt{\bar{\sigma}^{2} K^{2}+a^{2} c^{2} \cos ^{2} \phi}}$,

$\frac{\mathrm{d} K}{\mathrm{~d} \theta}=\left\{\begin{array}{ll}-3 B \cos 3 \theta & |\theta|>\theta_{\mathrm{T}} \\ -\sin \theta-\frac{1}{\sqrt{3}} \sin \phi \cos \theta & |\theta| \leq \theta_{\mathrm{T}}\end{array}\right.$,

$\frac{\partial \sigma_{\mathrm{m}}}{\partial \sigma}=\frac{1}{3}\left[\begin{array}{llllll}1 & 1 & 1 & 0 & 0 & 0\end{array}\right]^{\mathrm{T}}$,

$\frac{\partial \bar{\sigma}}{\partial \sigma}=\frac{1}{2 \bar{\sigma}}\left[\begin{array}{llllll}s_{x} & s_{y} & s_{z} & 2 \tau_{x y} & 2 \tau_{x z} & 2 \tau_{y z}\end{array}\right]^{\mathrm{T}}$,

$\frac{\partial J_{3}}{\partial \sigma}=\left\{\begin{array}{c}s_{y} s_{z}-\tau_{y z}^{2} \\ s_{x} s_{z}-\tau_{x z}^{2} \\ s_{x} s_{y}-\tau_{x y}^{2} \\ 2\left(\tau_{y z} \tau_{x z}-s_{z} \tau_{x y}\right) \\ 2\left(\tau_{x y} \tau_{y z}-s_{y} \tau_{x z}\right) \\ 2\left(\tau_{x z} \tau_{x y}-s_{x} \tau_{y z}\right)\end{array}\right\}+\frac{\bar{\sigma}^{2}}{3}\left\{\begin{array}{l}1 \\ 1 \\ 1 \\ 0 \\ 0 \\ 0\end{array}\right\}$.

Since the potential function $G$ has the same form as the yield function $F$, its differentiation with respect to stress $\left(\boldsymbol{n}^{\prime}\right)$ is similar to $\boldsymbol{n}$, except the angle of internal friction $\phi$ must be replaced with the dilation angle $\psi$.
As can be seen from the formulas listed above, when $|\theta|=30^{\circ}, C_{2}$ and $C_{3}$ are infinite values, resulting in the singularity of numerical calculation, and therefore, in the UMAT they are set to be zero when $|\theta|$ is greater than the transition angle $\theta_{\mathrm{T}}$.

\section{Stress adjustment in UMAT}

During incremental loading, stresses at some Gaussian integration points in a model may exceed yield stresses, accompanied with plastic strains, but the stresses of these points must be adjusted to the yield surface to meet the yield criterion and constitutive law. Thus, the backward Euler integral regression algorithm was adopted in the paper. The flowchart of the UMAT is shown in Fig. 3. Yield stress $\sigma_{y}$ varies with different yield criterions, and in the $\mathrm{M}-\mathrm{C}$ model it is defined as

$\sigma_{y}=\sigma_{y}^{0}+H^{\prime} \mathrm{d} \bar{\varepsilon}_{\mathrm{p}}=c \cos \phi+H^{\prime} \mathrm{d} \bar{\varepsilon}_{\mathrm{p}}$,

where $\mathrm{d} \bar{\varepsilon}_{\mathrm{p}}$ is the effective plastic strain; $H^{\prime}$ is the first derivative of hardening function $H$. Since no hardening or softening is considered in ideal elasto-plastic model, $H=H^{\prime}=0$, and $\sigma_{y}$ is constant.

Assuming that the stress vector of a Gaussian point is $\sigma^{r-1}$ after the $(r-1)$ th iteration, there are the following two cases on the adjustment of yield stress.

\subsection{First case}

As shown in Fig. 4, point $B$ has already yielded after the $(r-1)$ th iteration - that is to say, point $B$ is on the yield surface $F=0$ at this moment. Then at the $r$ th iteration the elastic trial stress $\sigma_{e}^{r}=\sigma^{r-1}+\mathrm{d} \sigma_{e}^{r}$ is calculated as segment $O A$. In other words, point $B$ moves to point $A$ due to obtaining the elastic stress increment calculated by $\mathrm{d} \sigma_{e}^{r}=$ $D_{e} \mathrm{~d} \varepsilon^{r}$ (segment $B A$ in Fig. 4) at the $r$ th iteration, in which $D_{e}$ is the elastic matrix. Point $A$ has been beyond the yield surface, but actually it is a stress point that an ideal elastoplastic material cannot reach. Therefore, to meet the yield criterion and constitutive relationship, point $A$ must be adjusted to point $D^{\prime}$ on the yield surface. Point $A$ cannot move back to point $B$ because a point can only move on the yield surface at yield state with the accompanying of plastic strain.

According to the vector diagram in Fig. 4, the stress calculation formula after adjustment is

$\sigma^{r}=\sigma^{r-1}+\mathrm{d} \sigma_{e}^{r}-\mathrm{d} D_{e} \mathrm{~d} \lambda$,

where $\mathrm{d} \lambda$ is the differential increment of the plastic multiplier $\lambda$, which can be expressed as 


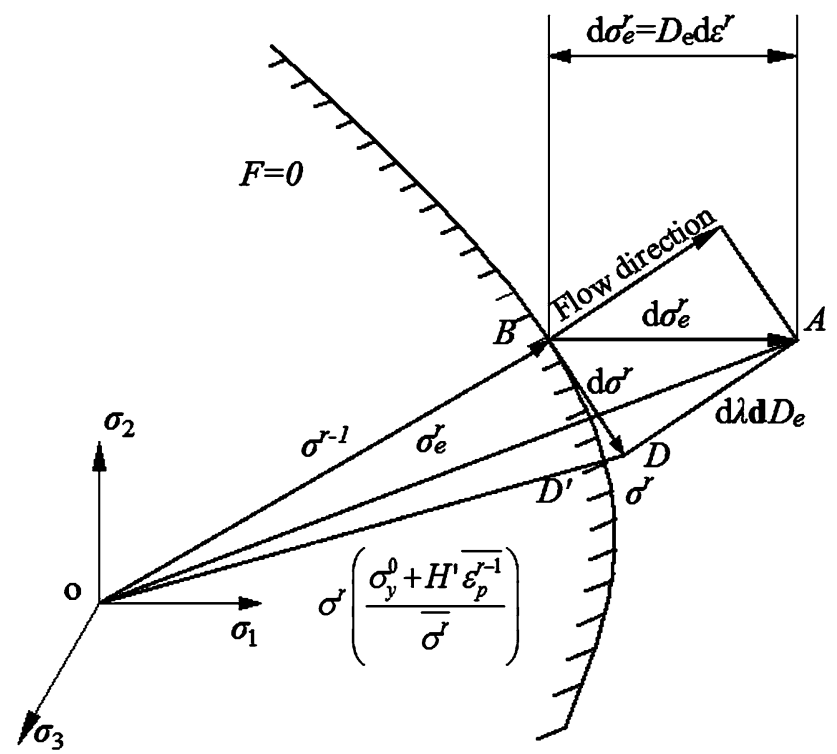

Fig. 4 Schematic of stress adjustment in the first case [19]

$\mathrm{d} \lambda=\frac{1}{H+n^{\mathrm{T}} \mathbf{d} D_{e}} \boldsymbol{n}^{\mathrm{T}} D_{e} \mathrm{~d} \varepsilon$,

where $\mathrm{d} D_{e}=D_{e} \boldsymbol{n}^{\prime}$.

By Eq. (13), point $A$ can only be adjusted to point $D$ (see Fig. 4) that may still deviate from point $D^{\prime}$. In order to move to point $D^{\prime}$, one may have

$O D \prime=k \cdot O D$,

where the adjustment coefficient $k$ is given by

$k=\frac{\sigma_{y}^{0}+H^{\prime} \bar{\varepsilon}_{\mathrm{p}}^{r}}{\bar{\sigma}^{r}}$,

where $\bar{\varepsilon}_{\mathrm{p}}^{r}$ is the effective plastic strain after the $r$ th iteration; $\bar{\sigma}^{r}$ is the effective stress given by

$\bar{\sigma}^{r}=\sigma_{\mathrm{m}} \sin \phi+\sqrt{\bar{\sigma}^{2} K^{2}(\theta)+a^{2} c^{2} \sin ^{2} \phi}$.

\subsection{Second case}

As shown in Fig. 5, a Gaussian point, for example point $C$, does not yield after the $(r-1)$ th iteration but it does at the $r$ th iteration. At the $(r-1)$ th iteration, point $C$ is inside the yield surface and in elastic state, but the elastic trial stress at the $r$ th iteration makes it move to point $A$, and $C A$ intersects with the yield surface at point $B$. The elastic stress increment calculated by $\sigma_{e}^{r}=D_{e} \mathrm{~d} \varepsilon^{r}$ (segment $C A$ ) at the $r$ th iteration involves two segments, i.e., $C B$ and $B A$, of which $C B$ is still inside the yield surface but $B A$ outside. Therefore, only segment $B A$ needs to be adjusted. That is to say, point $A$ must be adjusted back to $D^{\prime}$. From Fig. 5, one can see the stress value that needs to be adjusted is $R \mathrm{~d} \sigma_{e}^{r}$, in which the adjustment factor $R$ is

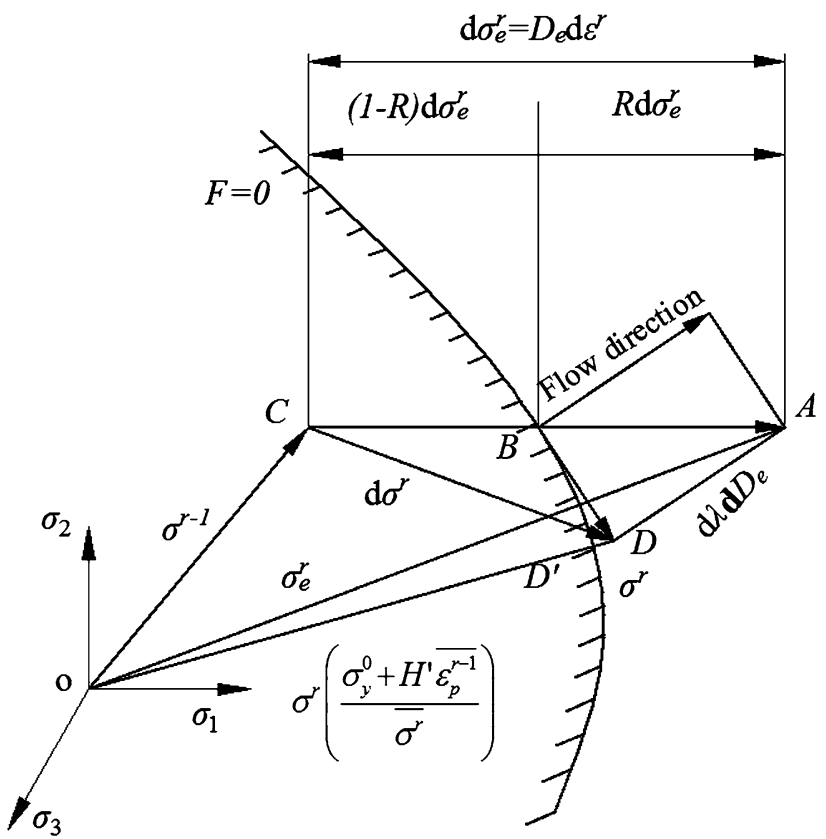

Fig. 5 Schematic of stress adjustment in the second case [19]

$R=\frac{B A}{C A}=\frac{\bar{\sigma}_{e}^{r}-\sigma_{y}}{\bar{\sigma}_{e}^{r}-\bar{\sigma}_{e}^{r-1}}$,

where $\sigma_{y}=\sigma_{0}=c \cdot \cos \phi$.

Equation (12) may still be used in stress adjustment. Similarly, point $A$ can merely be adjusted to point $D$. In order to move to point $D^{\prime}$, Eqs. (14) and (15) may still be used.

To sum up, in the process of finite element computation, stress adjustments are only conducted for yield Gaussian points in above two cases. Meanwhile, as is generally known, for those yield points, plastic strains accompanied with them can be calculated by the following orthogonal flow rule:

$\mathrm{d} \varepsilon^{\mathbf{p}}=\mathrm{d} \lambda \frac{\partial G}{\partial \boldsymbol{\sigma}}$.

Obviously, from Eq. (18), one can see that the flow direction of plastic strains is associated with the plastic potential function $G$. If the same dilation angle is considered, comparisons of the plastic potential surfaces determined by Eq. (9) in this paper and the potential surface proposed by Menétrey-William [16] and employed in the embedded $\mathrm{M}-\mathrm{C}$ model in ABAQUS are shown in Fig. 6. One can see that for any of the same strain Lode angle $\theta_{\varepsilon}$ except $\theta_{\varepsilon}= \pm 30^{\circ}$, the flow directions of plastic strains are different due to the difference of geometrical shapes of the two kinds of potential surfaces. Therefore, even if under the same loading condition after yielding, the total amount of plastic strains for both surfaces might be equal, each plastic strain component is not equal yet, such 


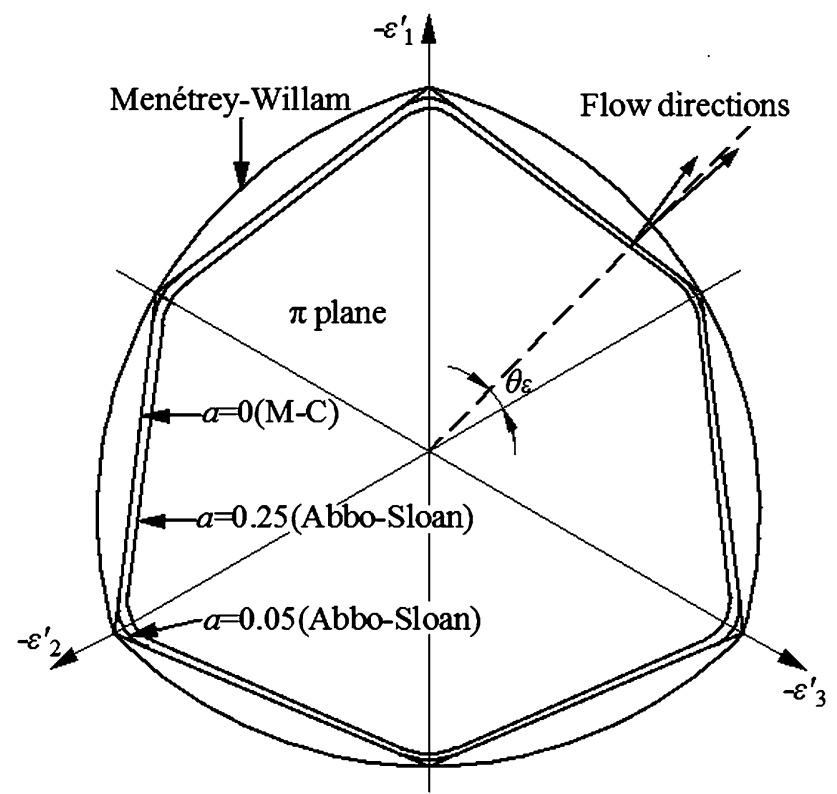

Fig. 6 Comparisons of plastic potential surfaces in $\pi$ plane in principal strain space

that the plastic shear and volumetric strains are different as well. Apparently, the potential surface used in this paper is a better approximation to the $\mathrm{M}-\mathrm{C}$ yield surface in shape, meaning that it is more reasonable than the one employed by the embedded $\mathrm{M}-\mathrm{C}$ model.

Based on the above theories, the UMAT subroutines were developed and their reliability will be validated in the following section by comparing with the embedded $\mathrm{M}-\mathrm{C}$ model in ABAQUS (hereafter refer to as ABAQUS) and the analytical solutions of the $\mathrm{M}-\mathrm{C}$ criterion.

\section{UMAT verification and application}

\subsection{Laboratory simulation tests}

A standard 3D model of a cylindrical soil specimen with a diameter of $39.1 \mathrm{~mm}$ and a height of $80 \mathrm{~mm}$ was built to numerically simulate the indoor conventional triaxial compression and uniaxial tensile tests. The model and its discretization are shown in Fig. 7. The cylindrical coordinates and C3D20R element type were adopted. The bottom in $Z$-axial direction and the side in $R$-axial direction were constrained, and the top was free. Self-weight stress was not considered in this modeling. The mechanical parameters of the clay were cited from Ref. [21], including the elastic modulus $E=2,300 \mathrm{kPa}$, the Poisson ratio $v=0.4$, the cohesion of the soil $c=21.43 \mathrm{kPa}$, the angle of internal friction $\phi=17.13^{\circ}$, and the unit weight $\gamma=17.8 \mathrm{kN} / \mathrm{m}^{3}$.

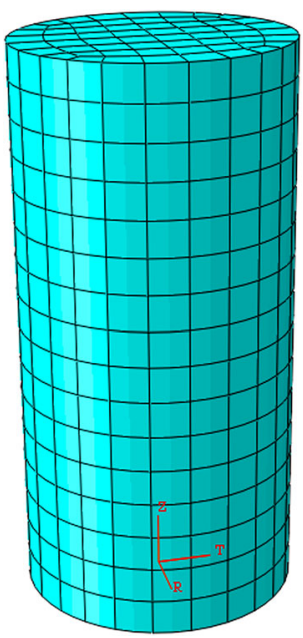

Fig. 7 3D finite element model and meshes of a standard specimen

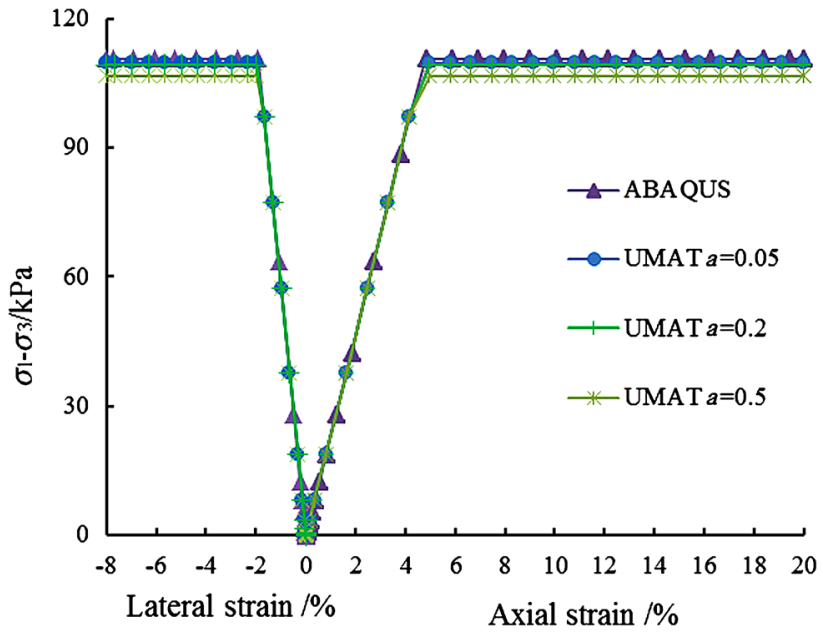

Fig. 8 Stress-strain curves of numerical simulations of triaxial compression

In the triaxial compression simulation, the model was loaded with a confining pressure of $63 \mathrm{kPa}$, and a downward displacement of $16 \mathrm{~mm}$ was applied on its top while in the uniaxial tensile simulation, without confining pressure, only an upward displacement of $5 \mathrm{~mm}$ was applied on its top.

Comparisons of the triaxial compression and uniaxial tests between the UMAT and ABAQUS model are shown in Figs. 8 and 9, respectively. It shows that in both the UMAT and ABAQUS model, the yield stresses are not affected by the magnitude of $\psi$. That is to say, the shear and tensile strengths of soil or rock are irrelevant to the associated and non-associated flow rules because the dilation angle $\psi$ does not occur to the yield functions, i.e., Eqs. (3) and (4), at all. 


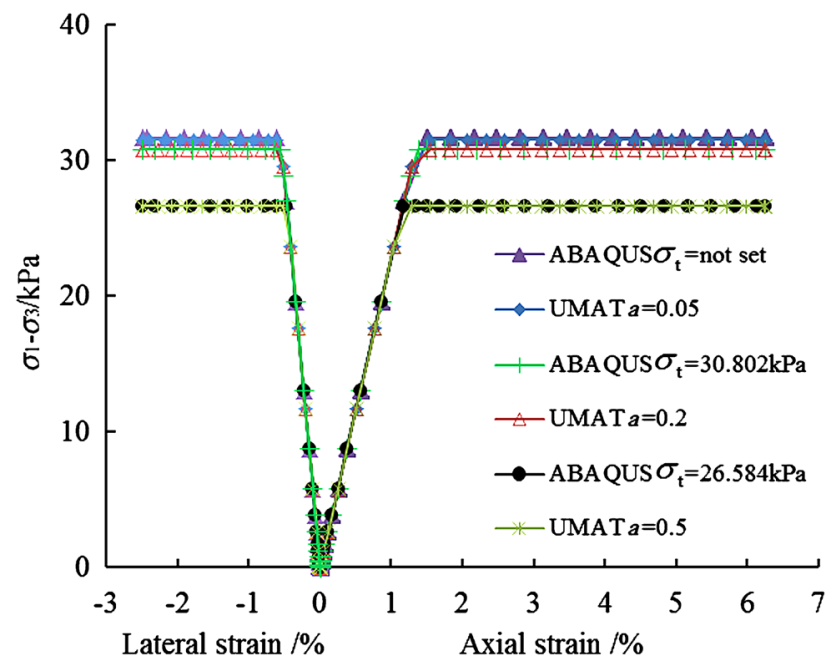

Fig. 9 Stress-strain curves of numerical simulations of uniaxial tension

As a matter of fact, the theoretical strengths for the triaxial compression and uniaxial tests can also be obtained by Eq. (2) or by drawing the Mohr circles (respectively see the biggest one and the first one on the right side of the $\tau$ axis in Fig. 10), which are 110.667 and $31.64 \mathrm{kPa}$, respectively. Almost the same results, i.e., 110.645 and $31.638 \mathrm{kPa}$, were respectively obtained by ABAQUS. It proves that the $\mathrm{M}-\mathrm{C}$ criterion is precisely replicated in the embedded M-C model in ABAQUS. Accordingly, when $a=0.05$, slightly less results, i.e., 109.674 and $31.506 \mathrm{kPa}$ (with the relative errors of $0.9 \%$ and $0.4 \%$ or so), were obtained by the UMAT, respectively. This is because the yield surface of Abbo-Sloan function is inside the $\mathrm{M}-\mathrm{C}$ yield surface, as can be seen in Figs. 1 and 2. However, evidently, when $a$ is less than or equal to 0.05 , the UMAT already can depict the $\mathrm{M}-\mathrm{C}$ criterion with a rather reliable precision. Despite of that, from Figs. 8 and 9, one can see that with the increase of $a$, the relative errors increase, particularly in the uniaxial tensile tests. And this law is still decided by the Abbo-Sloan hyperbolic yield function - the closer the maximum principal stress away from the tip of the $\mathrm{M}-\mathrm{C}$ yield surface, the greater the relative error.

Figure 10 also shows several special Mohr circles, such as the uniaxial compression $\left(\sigma_{1}>0, \sigma_{2}=\sigma_{3}=0\right)$, pure shear $\left(\sigma_{1}>0, \sigma_{2}=\sigma_{3}<0,\left|\sigma_{1}\right|=\left|\sigma_{2}\right|=\left|\sigma_{3}\right|\right)$, and triaxial tension circles $\left(\sigma_{1}>\sigma_{2} \geq \sigma_{3}>0\right)$. Note that the triaxial tension is one of the Mohr circles approaching the tip (point $O^{\prime}$ ) of the $\mathrm{M}-\mathrm{C}$ yield envelope. In line with the theory of the M-C criterion-Eq. (1), the possible maximum tensile stress $\sigma_{\text {tmax }}$ can be

$\sigma_{\operatorname{tmax}}=\frac{c}{\tan \phi}$.

That is to say, when $\sigma_{1}=\sigma_{2}=\sigma_{3}=\sigma_{\text {tmax }}=$ $69.53 \mathrm{kPa}$ (see Fig. 10), a stress point will reach point $O^{\prime}$ as far as this clay is concerned. To test the actual tensile strength, using the improved triaxial apparatus, Bishop and Garga had ever conducted the triaxial drained tension tests of the London clay [22], showing that under the low confining pressure range, i.e., $\sigma_{3}=-21$ to $-70 \mathrm{kPa}$, the tensile strength of the London clay is $\sigma_{1}=\sigma_{\text {tmax }}=27$ $34 \mathrm{kPa}$, which is much less than the theoretical strength determined by Eq. (19). Obviously, the loading conditions of their tests are similar to the two Mohr circles of tension under low confining pressures as shown in Fig. 11. It is easy to understand that without confining pressure, i.e., under the uniaxial tension, the tensile strength of clay should be less than those with low confining pressures and cannot reach the following theoretical strength expressed as Eq. (20):

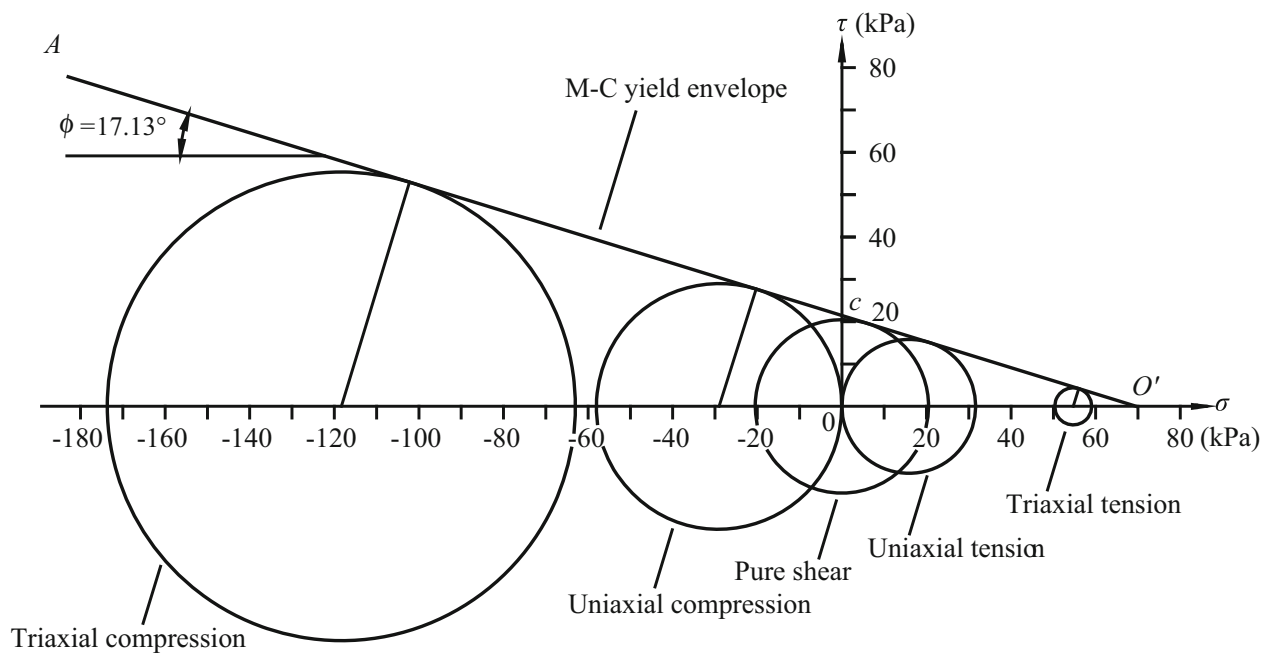

Fig. 10 Mohr circles corresponding to various stress states of soil 


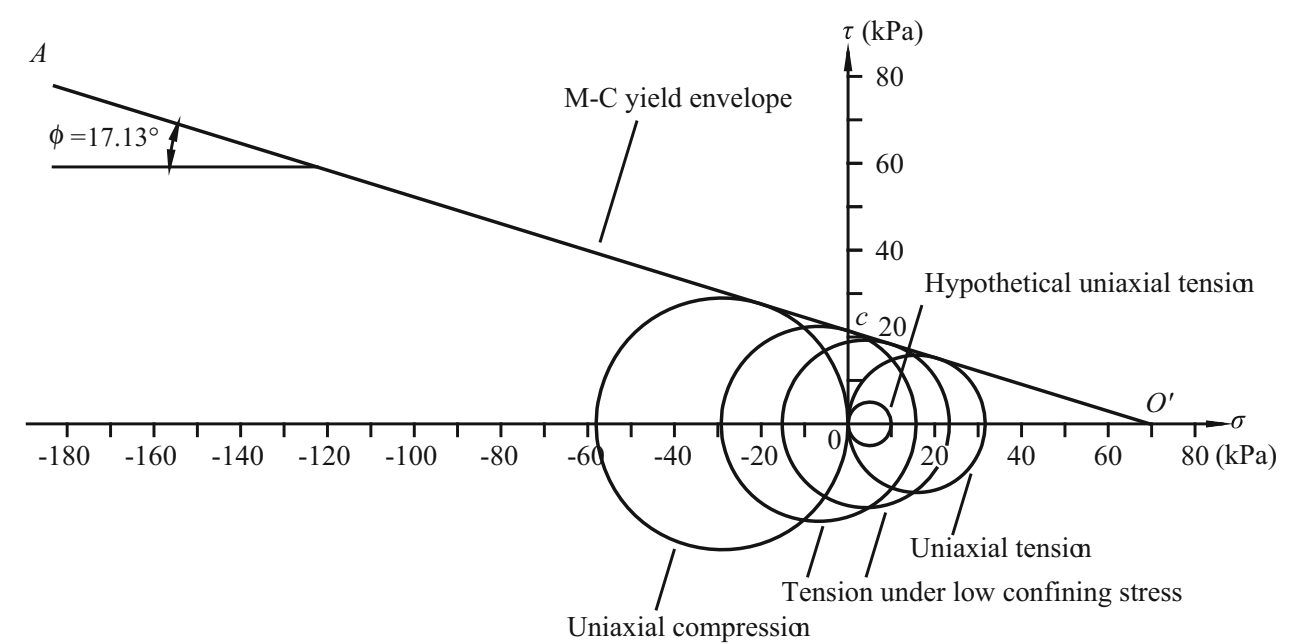

Fig. 11 Mohr circle analyses of tensile strengths of a soil

$\sigma_{\mathrm{t}}=\frac{2 c \cos \phi}{1+\sin \phi}$

derived from the $\mathrm{M}-\mathrm{C}$ shear criterion-Eq. (2), which is $31.64 \mathrm{kPa}$ exactly as far as the clay previously simulated is concerned. It is more impossible for clay to reach the maximum theoretical tensile strength determined by Eq. (19). In other words, the M-C shear yield criterion overestimates tensile strengths of soil and rock. Actually, when the major principal stress at a point of a soil or rock mass is positive or becomes tensile and exceeds the tensile strength of the soil or rock, the tensile rather than shear yield or failure will occur at this point.

For this reason, the tension cutoff can be considered by defining the uniaxial tensile strength of soil or rock when using the M-C model embedded in ABAQUS. Jia's comment (Ref. [9]) on that the tensile strengths of soil and rock are not considered in the embedded $\mathrm{M}-\mathrm{C}$ model is not pertinent. For the UMAT in this paper, tensile strength can be reflected by simply defining the magnitude of parameter $a$. That is to say, the value of $a$ decides not only the similarity between the Abbo-Sloan and M-C yield surfaces, but also the uniaxial tensile strength of soil or rock. As far as this clay is concerned, given $a=0.2$ or 0.5 , actually the uniaxial tensile strength was let be 30.802 or $26.584 \mathrm{kPa}$, and $\theta=-30^{\circ}$, of course, these two strength values can be obtained by Eq. (4) as well. Obviously, respectively specifying these two values to be the tension cutoff strengths, the precise numerical simulations can be realized by ABAQUS (see Fig. 9). If the uniaxial tensile strength of the soil is known or given by the Mohr circle of the hypothetical uniaxial tension as indicated in Fig. 11, then parameter $a$ can be calculated by Eq. (4).

\subsection{Stability analysis of a typical slope}

A homogeneous two-dimensional soil slope [17, 23, 24] was cited herein. The sizes and discretization of the model are shown in Fig. 12. The quadrilateral element with full integration (CPE4) was adopted to discretize the model. The grid density is completely consistent with those of Dawson et al. [17] and Fei and Zhang [24]. The mechanical parameters of the soil are the unit weight $\gamma=20 \mathrm{kN} / \mathrm{m}^{3}$, the cohesion $c=12.38 \mathrm{kPa}$, and the internal friction angle $\phi=20^{\circ}$. With these soil parameters, the stability safety factor of the slope is exactly 1.00 according to the limit analysis solution of Chen [23].

Over the last two decades or so, the finite element strength reduction technique has been widely used in slope stability analysis. But there is still a controversy on what judgment criterion should be adopted when determining

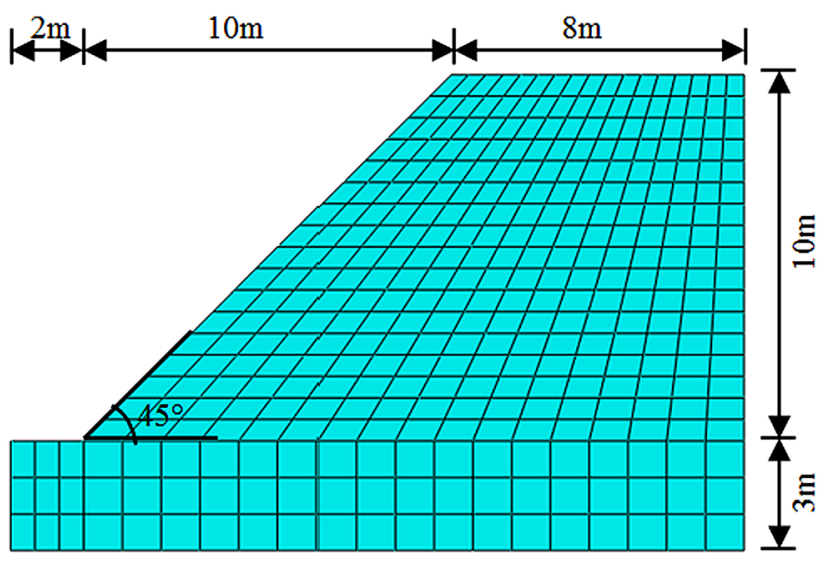

Fig. 12 Model sizes and meshes of a typical slope 
the factor $\left(F_{\mathrm{s}}\right)$ of safety. Generally, three judgment criteria were used: the non-convergence of numerical calculation (criterion 1), the plastic penetration (criterion 2), and the displacement mutation (criterion 3) [25-27]. Usually, the computed results by criteria 2 and 3 are not significant, and they are close to the results of the limit equilibrium or limit analysis methods. In this paper, the numerical analyses of the slope using criteria 1 and 2 were performed. When using the UMAT, the associated flow with $\psi=\phi=20^{\circ}$ and non-associated flow rules with $\psi=0.5 \phi=10^{\circ}$ or with $\psi=0.1^{\circ}$ (approximately $0^{\circ}$, which is tantamount to no consideration of dilation because $\psi$ is not allowed to equal zero in both ABAQUS and the UMAT to avoid numerical singularity) were adopted respectively. And let parameter $a$ be $0.05,0.5$, and 0.99 , respectively. Actually, when $a=0.5$ or 0.99 , it means the uniaxial tensile strength is 7.11 or around $0 \mathrm{kPa}$ according to Eq. (4). Here, two cases were considered. One is the conventional method that parameter $a$ of the whole soil mass is 0.5 or 0.99 . Usually, tensile stresses merely occur in the shallow layer near the top of a slope, so in the second case, for the deep layer, let $a$ be 0.05 , and for the shallow soil layer like the top three rows of elements in Fig. 12, let $a$ be 0.5 or 0.99 . Correspondingly, when using ABAQUS, firstly the maximum allowable dilation angle is $\psi=17.831^{\circ}$ to keep its potential surface convex and similar in shape to the $\mathrm{M}-\mathrm{C}$ yield surface. Then, when $\psi=10$ or $0.1^{\circ}$, similar analyses were performed with the tension cutoff of 7.11 and $0.01 \mathrm{kPa}$ (approximately $0 \mathrm{kPa}$ because it is not allowed to equal zero in ABAQUS to avoid numerical singularity). Only when the tension cutoff was considered, criterion 3 was adopted to determine the factors of safety because plastic penetration zone cannot be obtained. All the factors of safety are listed in Table 1.

From Table 1, the following conclusions can be drawn:

1. The factor of safety increases with the dilation angle, and it reaches the minimum (even less than 1.00) or maximum (greater than 1.00), respectively, when zero dilation or full dilation (associated flow rule) is considered.

2. When criterion 1 is used without tension cutoff, the computed factors of safety with respect to the three dilation angles by ABAQUS are respectively $15 \%$, $14 \%$, and $6 \%$ greater than the exact factor of safety of 1.00; when criterion 2 is used without tension cutoff, they are respectively $8 \%, 3 \%$ greater or $2 \%$ less than 1.00. Similar results are obtained by the UMAT. Moreover, for both ABAQUS and the UMAT, the factors of safety by criterion 1 often deviate from 1.00 farther than those of by criterion 2 .

3. When criterion 1 is used with tension cutoff, the computed factor of safety by ABAQUS obviously decreases but its variation with the dilation angle is unremarkable; when criterion 2 is used with the tension cutoff, ABAQUS is unable to show the plastic penetration zone so that no values are listed in those columns in Table 1, and in this case, criterion 3 was adopted to determine the factors of safety, which actually are around 0.94 for both tensile strengths $\sigma_{\mathrm{t}}=7.11$ and $0.01 \mathrm{kPa}$ and for all three dilation angles.

At this point, it shows that the computed results by ABAQUS in consideration of tension cutoff are not satisfying.

4. For the UMAT, when $a=0.05$ for the whole slope, which means that tensile yield is not considered, the factors of safety with criterion 2 are less than those by ABAQUS and closer to 1.00. The differences between the results from UMAT and ABAQUS are caused by the different potential functions.

5. The tensile strength of clay may be considered by the UMAT through changing the magnitude of parameter $a$, but comparisons show that merely in the potential tensile stress zone, $a$ set to be the value matched with the tensile strength is more reasonable, and if in slope stability analysis, $a$ is set to be 0.99 , that actually means no tensile but shear strength is considered.

Table 1 Computed factors of safety $F_{\mathrm{s}}$

\begin{tabular}{|c|c|c|c|c|c|c|c|}
\hline & & \multicolumn{2}{|c|}{$\psi=17.831^{\circ}$ for ABAQUS $\psi=20^{\circ}$ for UMAT } & \multicolumn{2}{|l|}{$\psi=10^{\circ}$} & \multicolumn{2}{|l|}{$\psi=0.1^{\circ}$} \\
\hline & & Criterion 1 & Criterion 2 & Criterion 1 & Criterion 2 & Criterion 1 & Criterion 2 \\
\hline \multirow[t]{3}{*}{ ABAQUS } & $\sigma_{\mathrm{t}}=$ not set & 1.15 & 1.08 & 1.14 & 1.03 & 1.06 & 0.98 \\
\hline & $\sigma_{\mathrm{t}}=7.11 \mathrm{kPa}$ & 1.02 & - & 1.02 & - & 0.98 & - \\
\hline & $\sigma_{\mathrm{t}}=0.01 \mathrm{kPa}$ & 0.96 & - & 0.96 & - & 0.95 & - \\
\hline \multirow[t]{4}{*}{ New UMAT } & $a=0.05$ & 1.07 & 1.04 & 1.07 & 1.02 & 0.99 & 0.97 \\
\hline & $a=0.5$ & 1.03 & 1.01 & 1.02 & 0.99 & 0.95 & 0.95 \\
\hline & $a=0.05$ or 0.5 & 1.05 & 1.03 & 1.05 & 1.01 & 0.98 & 0.97 \\
\hline & $a=0.05$ or 0.99 & 1.02 & 0.99 & 1.00 & 0.98 & 0.96 & 0.96 \\
\hline
\end{tabular}

" $\sigma_{\mathrm{t}}=$ not set" means that no tensile but shear failure was considered; "-" means that the shear plastic zone did not penetrate the whole slope 
Given the non-associated flow rule with no dilation $\left(\psi=0.1^{\circ}\right)$, without considering the tensile strength of this clay, Figs. 13 and 14 show the contours of the magnitude of equivalent plastic strains (PEMAG in ABAQUS or defined as SDV9 in the UMAT) and the contours of displacements in critical plastic penetration state, respectively, obtained by ABAQUS $\left(\sigma_{\mathrm{t}}=\right.$ not set) and the UMAT $(a=0.05)$. Evidently, the contour distributions and displacement magnitudes obtained by ABAQUS and the
UMAT are quite similar or close. The slight differences between them are attributed to the different potential functions. Likewise, without considering shear dilation but considering a uniaxial tensile strength of $7.11 \mathrm{kPa}$ (i.e., $\sigma_{\mathrm{t}}=7.11 \mathrm{kPa}$ in ABAQUS or for the whole slope, $a=0.5$ in the UMAT), the same comparisons are shown in Figs. 15 and 16. One can see that no plastic penetration zone is shown by ABAQUS even if non-convergence occurred, but it can still be revealed by the UMAT. To save
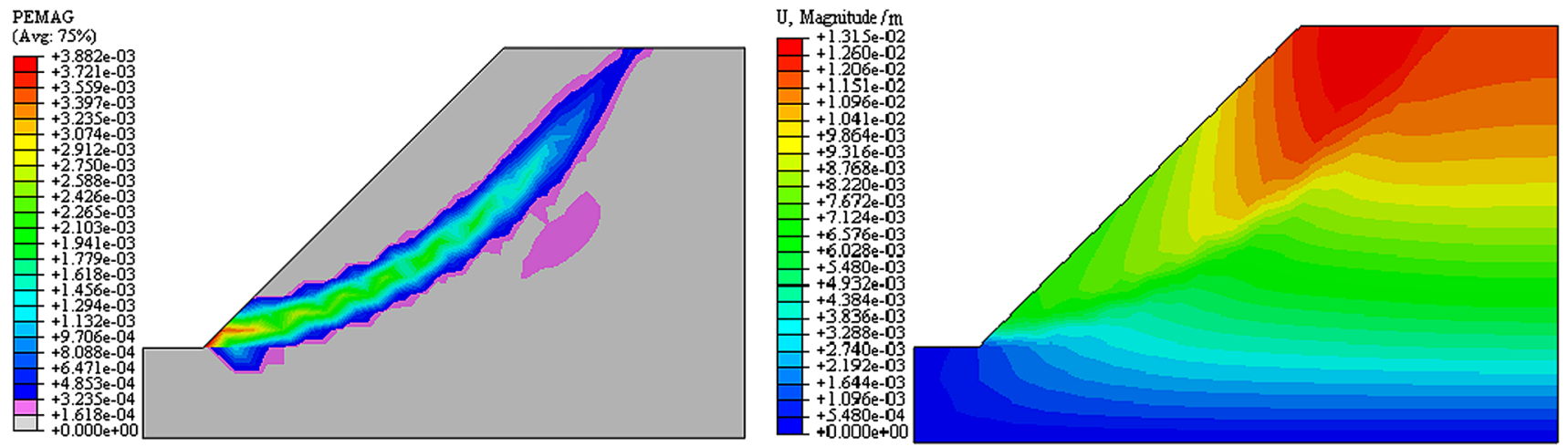

Fig. 13 Contours of PEMAG and displacements (ABAQUS, $\sigma_{\mathrm{t}}=$ not set)
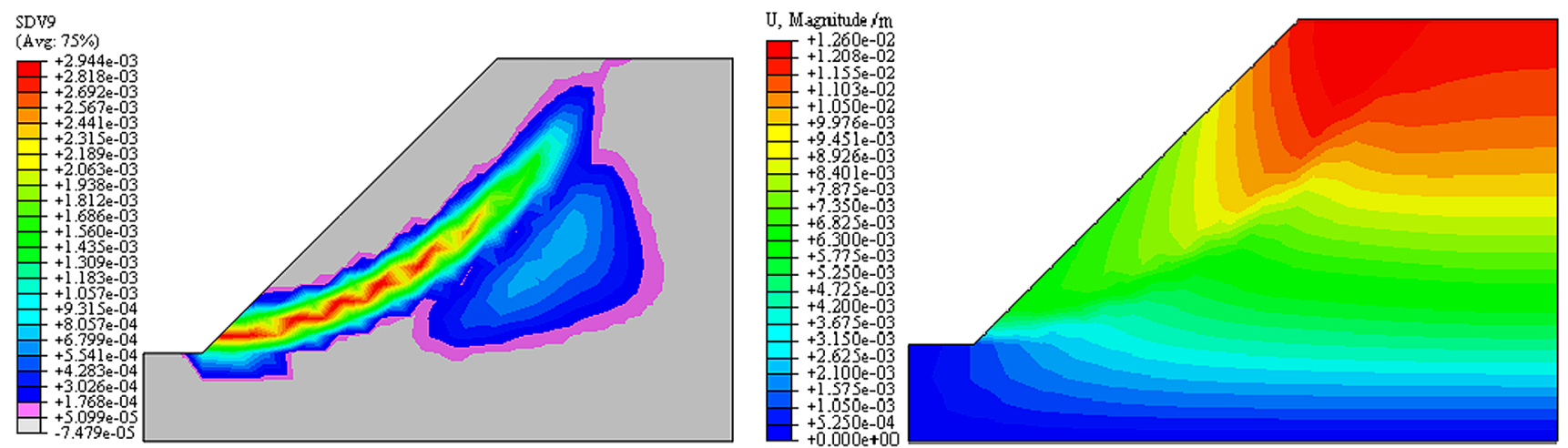

Fig. 14 Contours of PEMAG and displacements (UMAT, $a=0.05$ )
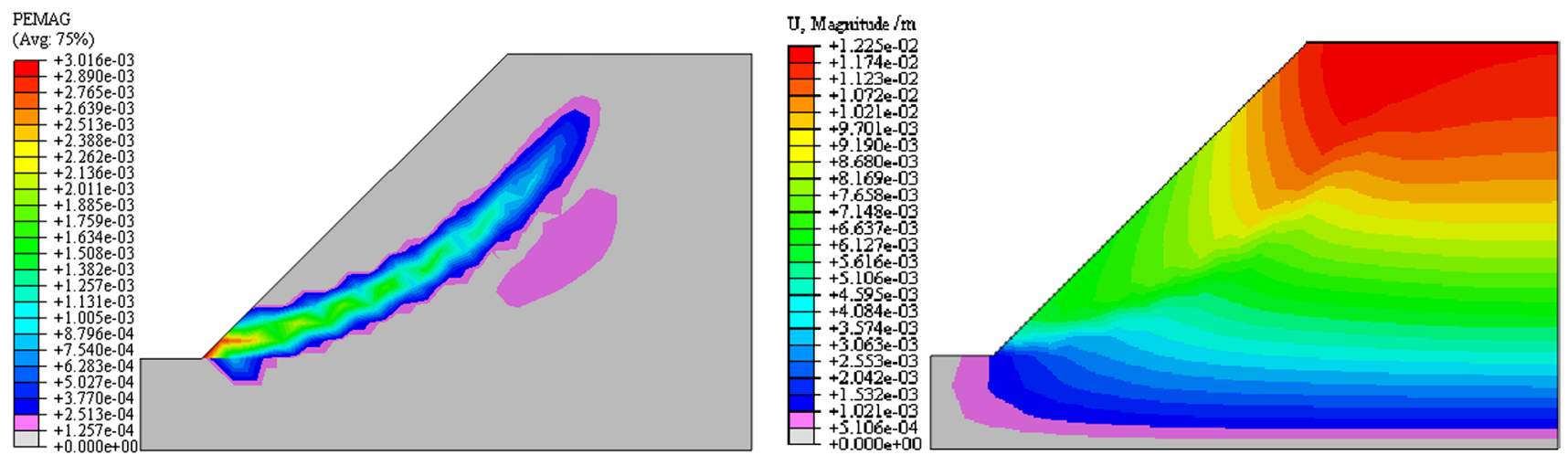

Fig. 15 Contours of PEMAG and displacements (ABAQUS, $\sigma_{\mathrm{t}}=7.11 \mathrm{kPa}, \psi=0.1^{\circ}$ ) 

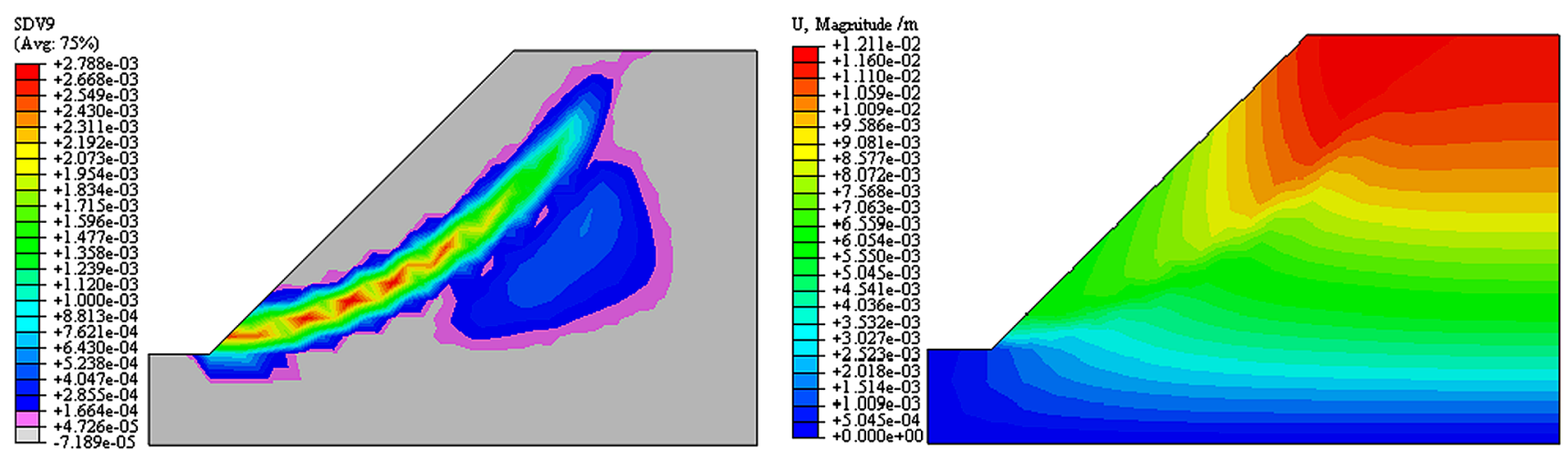

Fig. 16 Contours of PEMAG and displacements (UMAT, $a=0.5, \psi=0.1^{\circ}$ )

the space, the contour comparison of other cases were not provided here.

\section{Conclusions}

The modified Mohr-Coulomb model in this paper can approach the hexagonal pyramid surface when $\theta_{\mathrm{T}}=29^{\circ}$ and $a \leq 0.05$. The methods of stress adjustment after yielding avoid solving the second derivative of the plastic potential function and inverse matrix, and therefore, a fully implicit backward Euler integral regression algorithm was adopted in the UMAT and proved to be highly efficient in numerical convergence.

The examples of the numerical simulations on the triaxial compression and uniaxial tension tests show that both ABAQUS and the UMAT can obtain the solutions consistent with the analytical solutions. The magnitude of dilation angle does not affect the yield strength of soil or rock.

Theoretical analysis shows that the plastic flow direction is related to the potential function. Therefore, even if the total plastic strains are the same, the plastic strain components such as the plastic shear strain and the plastic volumetric strain may still be different due to different potential functions, which have been proven by comparisons of the contours of equivalent plastic strains and displacements obtained by ABAQUS and the UMAT.

Comparisons of the typical soil slope stability analyses by ABAQUS and the UMAT show that the differences are not remarkable when only the shear yield and the plastic penetration criterion are considered. The results by the UMAT are slightly less than that by ABAQUS and closer to the theoretical results. Therefore, the UMAT is more reliable than the embedded Mohr-Coulomb model.

The factor of safety of a slope is influenced by the dilation angle. The larger the dilation angle, the greater the factor of safety, and the factor of safety reaches its maximum when the associated flow rule (the dilation angle equals to the angle of internal friction of soil or rock) is adopted by the UMAT. Usually, the limit equilibrium method and limit analysis method cannot fully consider the influence of the dilation of soil and rock. Consequently, if the accurate value of the dilation angle of soil or rock can be obtained by test, the results by the UMAT or ABAQUS will be definitely more reliable than those of the limit equilibrium and limit analysis methods.

When the tensile strength of soil or rock is considered, it seems the factor of safety by ABAQUS is not so reliable because it is not sensitive to the magnitude of tensile strength. The relatively accurate results, however, can still be obtained by the UMAT through setting parameter $a$ in the potential tensile stress zone of a slope to be the value matched with the tensile strength.

Open Access This article is distributed under the terms of the Creative Commons Attribution 4.0 International License (http:// creativecommons.org/licenses/by/4.0/), which permits unrestricted use, distribution, and reproduction in any medium, provided you give appropriate credit to the original author(s) and the source, provide a link to the Creative Commons license, and indicate if changes were made.

\section{References}

1. Zienkiewicz OC, Taylor RL (2000) The finite element method. Solid mechanics, vol 2, 5th edn. Woburn, Butterworth Heinemann

2. Drucker DC, Prager W (1952) Soil mechanics and plastics analysis or limit design. Quat Appl Math 10(2):157-165

3. Alejano LR, Bobet A (2012) ISRM suggested method: DruckerPrager Criterion. Rock Mech Rock Eng 45:995-999

4. Deng CJ, He GJ, Zheng YR (2006) Studies on Drucker-Prager yield criterions based on $\mathrm{M}-\mathrm{C}$ yield criterion and application to geotechnical engineering. Chin J Geotech Eng 28(6):735-739 (in Chinese)

5. Dai ZH, Shen PS (2003) Simplified form and applications of Mohr-Coulomb equivalent area circle yield criterion. J Fuzhou Univ 31(4):454-459 (in Chinese)

6. Koiter WT (1953) Stress-strain relations, uniqueness and variational theorems for elastic-plastic materials with a singular yield surface. Quart Appl Math 11:350-354 
7. Clausen J, Damkilde L, Andersen L (2006) Efficient return algorithms for associated plasticity with multiple yield surfaces. Int J Numer Meth Eng 66(6):1036-1059

8. Abbo AJ, Sloan SW (1995) A smooth hyperbolic approximation to the Mohr-Coulomb yield criterion. Comput Struct 54(3):427-441

9. Jia SP, Chen WZ, Yang JP, Chen PS (2010) An elastoplastic constitutive model based on modified Mohr-Coulomb criterion and its numerical implementation. Rock and Soil Mechanics 31(7):2051-2058 (in Chinese)

10. Peng MX, Chen J (2013) Coulomb's solution to seismic passive earth pressure on retaining walls. Can Geotech J 50(10):1100-1107

11. Peng MX, Chen J (2013) Slip-line solution to active earth pressure on retaining walls. Geotechnique 63(12):1008-1019

12. Xu LY, Cai F, Wang GX, et al (2013) Nonlinear analysis of laterally loaded single piles in sand using modified strain wedge model. Comput Geotech 51:60-71

13. Lee YK, Pietruszczak S, Choi BH (2012) Failure criteria for rocks based on smooth approximations to Mohr-Coulomb and Hoek-Brown failure functions. Int $J$ Rock Mech Min Sci 56:146-160

14. Sven K, Lars D, Kristian K (2012) Lower-bound calculations of the bearing capacity of eccentrically loaded footings in cohesionless soil. Can Geotech J 49(3):298-310

15. Dimitrios L, Tanusree C, Rodrigo S (2006) Bearing capacity of strip footings on purely frictional soil under eccentric and inclined loads. Can Geotech J 45(6):768-787
16. Menétrey PH, William KJ (1995) Triaxial failure criterion for concrete and its generalization. ACI Struct J 92(3):311-318

17. Dawson EM, Roth WH, Drescher A (1999) Slope stability analysis by strength reduction. Geotechnique 49(6):835-840

18. Dassault Systèmes Simulia Corp (2012) ABAQUS Analysis User's Manual

19. Owen DRJ, Hinton E (1980) Finite elements in plasticity theory and practice. Pinerige press limited

20. Abbo AJ, Lyamin AV, Sloan SW et al (2011) A C2 continuous approximation to the Mohr-Coulomb yield surface. Int J Solids Struct 48(21):3001-3010

21. Chen LJ, Dai ZH, Liu ZW (2011) Three-dimensional nonlinear finite element analysis of soft soil excavation engineering considering $\mathrm{K}_{0}$ consolidation. Rock Soil Mech 32(12):3796-3804 (in Chinese)

22. Bishop AW, Garga VK (1969) Drained tension tests on Londonclay. Geotechnique 9(2):309-313

23. Chen WF (1975) Limit analysis and soil plasticity. Elsevier, Amsterdam

24. Fei K, Zhang JW (2010) Application of ABAQUS in geotechnical engineering. China waterpower press, Peking (in Chinese)

25. Griffiths DV, Lane PA (1999) Slope stability analysis by finite elements. Geotechnique 49(3):387-403

26. Ren S, Beley JD, Mukherjee S et al (2006) Probabilistic finite element analysis using ANSYS. Struct Saf 28(1-2):17-43

27. Eiramly H, Morgenstern NR, Cruden DM (2005) Probabilistic assessment of stability of cut slope in residual soil. Geotechnique 55(1):77-84 